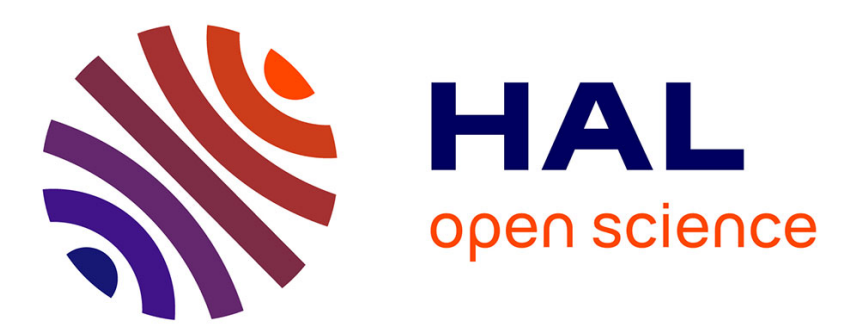

\title{
Dyskinésies ciliaires primitives : actualités diagnostiques
}

S. Blanchon, M. Legendre, A. Coste, N. Beydon, S. Amselem, Estelle

Escudier, J.-F. Papon

\section{To cite this version:}

S. Blanchon, M. Legendre, A. Coste, N. Beydon, S. Amselem, et al.. Dyskinésies ciliaires primitives : actualités diagnostiques. Revue francaise d'allergologie, 2016, 56 (3), pp.209-211. 10.1016/j.reval.2016.01.014 . hal-01302495

\section{HAL Id: hal-01302495 \\ https://hal.sorbonne-universite.fr/hal-01302495}

Submitted on 14 Apr 2016

HAL is a multi-disciplinary open access archive for the deposit and dissemination of scientific research documents, whether they are published or not. The documents may come from teaching and research institutions in France or abroad, or from public or private research centers.
L'archive ouverte pluridisciplinaire HAL, est destinée au dépôt et à la diffusion de documents scientifiques de niveau recherche, publiés ou non, émanant des établissements d'enseignement et de recherche français ou étrangers, des laboratoires publics ou privés. 


\section{Dyskinésies ciliaires primitives : actualités diagnostiques}

Sylvain Blanchon, Marie Legendre, André Coste, Nicole Beydon, Serge Amselem, Estelle Escudier*, Jean-François Papon

\section{$\underline{\text { Affiliations }}$}

Sylvain Blanchon (PH), Unité de pneumologie et allergologie pédiatrique, Hôpital des Enfants, Centre Hospitalier Universitaire, Toulouse. blanchon.s@chu-toulouse.fr

Marie Legendre (PH), Université Pierre et Marie Curie, UMR_S933, Service de Génétique et Embryologie Médicales, Hôpital Armand-Trousseau, Paris. marie.legendre@aphp.fr

André Coste (PU-PH), Service d'ORL et de Chirurgie Cervico-Faciale, Centre Hospitalier Intercommunal de Créteil, Créteil. andre.coste@ chicreteil.fr

Nicole Beydon (PH), Service de physiologie. Unité fonctionnelle d'explorations respiratoires, Hôpital Armand-Trousseau, Paris. nicole.beydon@aphp.fr

Serge Amselem (PU-PH), Université Pierre et Marie Curie, UMR_S933, Service de Génétique et Embryologie Médicales, Hôpital Armand-Trousseau, Paris. $\underline{\text { serge.amselem@aphp.fr }}$

Estelle Escudier (MCU-PH), Université Pierre et Marie Curie, UMR_S933, Service de Génétique et Embryologie Médicales, Hôpital Armand-Trousseau, Paris. estelle.escudier@aphp.fr 
Jean-François Papon (MCU-PH), Service d'ORL et de Chirurgie Cervico-Faciale, Centre Hospitalier Universitaire, Le Kremlin-Bicêtre. jean-francois.papon@aphp.fr

Mots clé : cil, battement ciliaire, microscopie électronique, monoxyde d'azote, génétique

\section{Auteur correspondant :}

*Estelle Escudier

Service de Génétique et Embryologie Médicales (Pr S Amselem)

Hôpital Armand-Trousseau,

26 avenue Arnold Netter

75571 Paris Cedex 12

Tél : $0144735244 \quad$ Fax : 0144735219

estelle.escudier@aphp.fr 
Les dyskinésies ciliaires primitives (DCP) sont des affections génétiques rares, liées à une anomalie de structure et/ou de fonction des cils. Il existe 2 types de cils : les cils mobiles des cellules multiciliées qui transportent fluides ou cellules; les cils primaires, uniques pour chaque cellule et immobiles (en dehors des cils des cellules embryonnaires nodales) qui sont des récepteurs sensibles aux modifications de l'environnement cellulaire. Les DCP sont responsables d'infections chroniques des voies aériennes, débutant dans l'enfance, et évoluant vers des dilatations des bronches. Le défaut de mobilité ciliaire des cellules embryonnaires nodales entraine une perturbation du flux du liquide amniotique normalement orienté vers la gauche du disque embryonnaire. Il s'en suit une latéralisation aléatoire des organes et la survenue d'un situs inversus chez $\sim 50 \%$ des patients, réalisant alors le syndrome de Kartagener (bronchectasies, situs inversus et sinusite chronique).[1]

\section{Structure des cils mobiles}

L'axonème, cytosquelette interne des cils et des flagelles des spermatozoïdes conservé au cours de l'évolution, comporte 9 doublets de microtubules périphériques disposés autour d'une paire de microtubules centraux entourés d'une gaine (complexe central). Les doublets sont reliés entre eux par les liens de nexine-complexes de régulation des dynéines (Dynein regulatory complex, DRC) et au complexe central par les ponts radiaires. Les bras de dynéine sont des complexes multiprotéiques qui possèdent l'activité ATPasique indispensable au battement ciliaire. [2]

\section{Mouvement ciliaire}

Le battement du cil se décompose en une phase active brève pendant laquelle, le cil propulse le mucus, suivie d'une phase de récupération où le cil recourbé revient à sa position initiale. La fréquence de battement ciliaire (FBC) est physiologiquement de 10 à 15Hz. [3] 


\section{Anomalies de l'ultrastructure ciliaire}

Le spectre des anomalies décrites dans les DCP montrent que les bras de dynéine sont le plus souvent concernés ( $\sim 50 \%$ des cas), plus rarement il existe des anomalies du complexe central, enfin, chez certains patients, aucune anomalie ultrastructurale n'est identifiée [4].

\section{Génétique des DCP}

L'incidence des DCP est estimée à 1/16 000 naissance, mais elle est probablement plus élevée étant donné l'existence de formes frustres, non diagnostiquées. La transmission des DCP se fait selon un mode autosomique récessif. L'hétérogénéité génétique a d'emblée été évoquée sur la diversité des anomalies ultrastructurales. DNAII a été le premier gène identifié en 1999 [5], et à ce jour 31 gènes ont été impliqués dans la DCP. Ces gènes codent pour des protéines de structure, d'assemblage ou régulant le mouvement ciliaire. Deux autres gènes, $M C I D A S$ et $C C N O$, sont responsables d'un défaut de ciliogenèse de présentation similaire aux DCP [Table 1] [2]. L'anomalie ultrastructurale identifiée permet d'orienter le groupe de gènes qui doivent être analysés. Actuellement, des mutations non ambiguës permettent de confirmer le diagnostic de DCP chez plus de la moitié des patients.

\section{Démarche diagnostique}

En cas de symptomatologie évocatrice de DCP et après élimination des diagnostics différentiels, les tests fonctionnels (mesure du monoxyde d'azote (NO) nasal et étude du mouvement ciliaire) permettent de sélectionner les patients pour étude de l'ultrastructure ciliaire afin de confirmer le diagnostic de DCP et de guider l'étude génétique [6].

\section{NO nasal}

Le NO est un gaz produit au niveau rhino-sinusien à des valeurs très hautes et à un niveau moindre au niveau alvéolaire et bronchique. Les valeurs de NO nasal mesurées chez les patients atteints de DCP sont remarquablement plus basses $(<100 \mathrm{~nL} / \mathrm{min})$ que celles 
mesurées chez les sujets sains (>250 nL/min) ou atteints de bronchopathies chroniques d'autres étiologies. [7,8]

\section{Mouvement ciliaire}

L'étude du mouvement ciliaire est réalisée à partir de cellules ciliées obtenues par brossage nasal et/ou bronchique effectué à distance d'un épisode infectieux (>1 mois).

Le mouvement ciliaire peut être évalué de manière semi-quantitative en microscopie optique, mais cette analyse est subjective et imprécise [6]. La vidéomicroscopie à haute vitesse (VMHV) (120 à 350 images/seconde), couplant une caméra numérique à un microscope optique, visualise au ralenti le mouvement des cils. Elle permet non seulement de mesurer la FBC, mais aussi de comparer les phases active et passive et d'évaluer la coordination des cils. Des paramètres objectifs d'analyse du mouvement ont été développés améliorant la sensibilité et la spécificité de la VMHV : longueur des cils, amplitude du mouvement, temps de pose, distance parcourue par la pointe du cil ou surface balayée par le cil [9]. Enfin, la forme du mouvement ciliaire de patients atteints de DCP serait caractéristique de chaque anomalie ultrastructurale [10]. En 2009, la Task Force de l'ERS a recommandé l'utilisation de la VMHV pour l'analyse du mouvement ciliaire [6].

\section{Ultrastructure ciliaire}

L'ultrastructurale ciliaire est analysée selon les techniques classiques de microscopie électronique à transmission, sur des biopsies nasale ou bronchique. Les résultats sont exprimés de manière quantitative (\% de cils anormaux/nombre de cils étudiés) et qualitatifs (type de l'anomalie dominante, anomalie unique ou polymorphe). Chez les sujets normaux, il existe un fond d'anomalies ciliaires ( $5 \%)$ portant essentiellement sur le nombre de microtubules. Chez les patients atteints de DCP, la même anomalie ciliaire touche tous les cils (sauf pour les anomalies du complexe central qui concernent moins de $50 \%$ des cils). Chez les hommes atteints de DCP, l'anomalie flagellaire des spermatozoïdes est identique à celle des cils. [4]. 


\section{Génétique}

Le nombre élevé et la taille souvent grande des gènes des DCP ne permettent pas de réaliser de première intention des analyses moléculaires à visée diagnostique. Le choix des gènes à étudier est guidé par le défaut de l'ultrastructure ciliaire. La mise en place du séquençage à haut débit (panel ciblé des gènes de DCP par NGS, Next Generation Sequencing) devrait permettre de progresser dans le diagnostic moléculaire des DCP.

\section{Conclusion et perspectives}

La confirmation diagnostique de la DCP reste souvent difficile et très spécialisée. Elle repose sur l'affirmation d'anomalies de mouvement et/ou de structure des cils respiratoires. En 2016, l'ERS devrait publier des recommandations européennes basées sur les preuves pour le diagnostic des DCP afin d'uniformiser et de diffuser les bonnes pratiques. 


\section{BIBLIOGRAPHIE}

1. Afzelius BA. A human syndrome caused by immotile cilia. Science, 1976, 183:317-319

2. Praveen K, Davis EE, Katsanis N. Unique among ciliopathies: primary ciliary dyskinesia, a motile cilia disorder. F1000Prime Rep, 2015, $10: 7: 36$

3. - Knowles MR, Boucher RC. Mucus clearance as a primary innate defense mechanism for mammalian airways. J Clin Invest, 2002, 109(5):571-7.

4. Papon JF, Coste A, Roudot-Thoraval F et al. A 20-year experience of electron microscopy in the diagnosis of primary ciliary dyskinesia. Eur Respir J, 2010, 35(5):1057-63

5. Pennarun G, Escudier E, Chapelin C et al. Loss-of-function mutations in a human gene related to Chlamydomonas reinhardtii dynein IC78 result in primary ciliary dyskinesia. Am J Hum Genet, 1999, 65:1508-15019

6. Barbato A, Frischer T, Kuehni CE et al. Primary ciliary dyskinesia: a consensus statement on diagnostic and treatment approaches in children. Eur Respir J, 2009, 34(6):1264-76

7. Marthin JK, Nielsen KG. Choice of nasal nitric oxide technique as first-line test for primary ciliary dyskinesia. Eur Respir J, 2011, 37(3):559-65

8. Narang I, Ersu R, Wilson NM, Bush A. Nitric oxide in chronic airway inflammation in children: diagnostic use and pathophysiological significance. Thorax, 2002, 57(7):586-589.

9. Papon JF, Bassinet L, Cariou-Patron G et al. Quantitative analysis of ciliary beating in primary ciliary dyskinesia: a pilot study. Orphanet J Rare Dis, 2012, 7:78-89

10. Chilvers MA, O'Callaghan C. Analysis of ciliary beat pattern and beat frequency using digital high speed imaging: comparison with the photomultiplier and photodiode methods. Thorax, 2000, 55(4):314-7 
Table 1 : Etiologies moléculaires des DCP (31 gènes) et des défauts de ciliogénèse (2 gènes), en fonction du type d'anomalie de l'ultrastructure ciliaire (AR : autosomique récessif).

\begin{tabular}{|c|c|c|}
\hline & Gène & Mode de transmission \\
\hline Absence des bras de dynéine externes & $\begin{array}{l}\text { ARMC4 } \\
C C D C 114 \\
C C D C 151 \\
D N A H 5 \\
\text { DNAI1 } \\
\text { DNAI2 } \\
\text { DNAL1 } \\
\text { NME8 (TXNDC3) }\end{array}$ & $\begin{array}{l}\text { AR } \\
\text { AR } \\
\text { AR } \\
\text { AR } \\
\text { AR } \\
\text { AR } \\
\text { AR } \\
\text { AR }\end{array}$ \\
\hline $\begin{array}{l}\text { Absence des bras de dynéine externes } \\
\text { et internes }\end{array}$ & $\begin{array}{l}\text { C21orf59 } \\
\text { CCDC103 } \\
\text { DNAAF1 (LRRC50) } \\
\text { DNAAF2 (KTU) } \\
\text { DNAAF3 } \\
\text { DYX1C1 (DNAAF4) } \\
\text { DNAAF5 (HEATR2) } \\
\text { LRRC6 } \\
\text { RPGR } \\
\text { SPAG1 } \\
\text { ZMYND10 }\end{array}$ & $\begin{array}{l}\text { AR } \\
\text { AR } \\
\text { AR } \\
\text { AR } \\
\text { AR } \\
\text { AR } \\
\text { AR } \\
\text { AR } \\
\text { Récessif lié à l'X } \\
\text { AR } \\
\text { AR }\end{array}$ \\
\hline $\begin{array}{l}\text { Absence des bras de dynéine internes } \\
\text { et désorganisation axonémale }\end{array}$ & $\begin{array}{l}C C D C 39 \\
C C D C 40\end{array}$ & $\begin{array}{l}\text { AR } \\
\text { AR }\end{array}$ \\
\hline $\begin{array}{l}\text { Défauts du complexe de régulation } \\
\text { des dynéines }\end{array}$ & $\begin{array}{l}C C D C 65 \\
D R C 1(C C D C 164) \\
G A S 8\end{array}$ & $\begin{array}{l}\text { AR } \\
\text { AR } \\
\text { AR }\end{array}$ \\
\hline Anomalies du complexe central & $\begin{array}{l}H Y D I N \\
\text { RSPH1 } \\
\text { RSPH3 } \\
\text { RSPH4A } \\
\text { RSPH9 }\end{array}$ & $\begin{array}{l}\text { AR } \\
\text { AR } \\
\text { AR } \\
\text { AR } \\
\text { AR }\end{array}$ \\
\hline Ultrastructure normale & DNAH11 & AR \\
\hline Non connue & DNAH8 & AR \\
\hline Défaut de ciliogénèse & $\begin{array}{l}\text { CCNO } \\
M C I D A S\end{array}$ & $\begin{array}{l}\mathrm{AR} \\
\mathrm{AR}\end{array}$ \\
\hline
\end{tabular}

